05

\title{
Аномальный эффект Холла в фрустрированных магнетиках
}

\author{
(C) В.В. Глушков ${ }^{1,2}$, М.А. Анисимов ${ }^{1}$, А.В. Богач ${ }^{1,5}$, А.Д. Божко ${ }^{1}$, С.В. Демишев ${ }^{1,2}$, \\ В.Н. Краснорусский ${ }^{1}$, А.Н. Самарин ${ }^{1,3}$, В.Б. Филипов ${ }^{4}$, Н.Ю. Шицевалова ${ }^{4}$ \\ ${ }^{1}$ Институт общей фризики им. А.М. Прохорова РАН, \\ Москва, Россия \\ ${ }^{2}$ Национальный исследовательский университет „Высшая школа экономики“, \\ Москва, Россия \\ ${ }^{3}$ Московский фризико-технический институт (государственный университет), \\ Долгопрудный, Россия \\ ${ }^{4}$ Институт проблем материаловедения им. И. Францевича НАНУ, \\ Киев, Украина \\ ${ }^{5}$ Национальный исследовательский технологический университет „МИСИС“, \\ Москва, Россия \\ E-mail: glushkov@lt.gpi.ru
}

Поступила в Редакцию 15 апреля 2019 г.

В окончательной редакции 22 апреля 2019 г.

Принята к публикации 24 апреля 2019 г.

Для твердых растворов замещения $\mathrm{Ho}_{0.5} \mathrm{Lu}_{0.5} \mathrm{~B}_{12}, \mathrm{Mn}_{1-x} \mathrm{Fe}_{x} \mathrm{Si}(0 \leq x \leq 1)$ и $\mathrm{Eu}_{1-x} \mathrm{Gd}_{x} \mathrm{~B}_{6}(x<0.04)$ выполнен сравнительный анализ эффекта Холла в диапазоне температур $2-300 \mathrm{~K}$ в магнитных полях до $8 \mathrm{~T}$. Для систем с различными видами магнитной фрустрации идентифицирован аномальный вклад в эффект Холла $\rho_{x y}^{A} \sim \rho_{x x} M$. Линейный скейлинг $\rho_{x y}^{A} \sim \rho_{x x}$, зарегистрированный в диапазоне сопротивлений $\rho_{x x} \sim 0.01-1 \mathrm{~m} \Omega \cdot \mathrm{cm}$ вне области применимости классической модели асимметричного рассеяния, связывается с ростом амплитуды спиновых флуктуаций в парамагнитной фазе исследуемых соединений с неколлинеарной магнитной структурой. Для $\mathrm{Ho}_{0.5} \mathrm{Lu}_{0.5} \mathrm{~B}_{12}$ и $\mathrm{Eu}_{1-x} \mathrm{Gd}_{x} \mathrm{~B}_{6}$ выделен топологический вклад в эффект Холла, амплитуда которого варьируется от $80 \mathrm{n} \Omega \cdot \mathrm{cm}$ (для $\mathrm{Ho}_{0.5} \mathrm{Lu}_{0.5} \mathrm{~B}_{12}$ ) до $7.5 \mu \Omega \cdot \mathrm{cm}$ (для $\left.\mathrm{Eu}_{0.97} \mathrm{Gd}_{0.03} \mathrm{~B}_{6}\right)$.

Ключевые слова: аномальный эффект Холла, фрустрация, антиферромагнетизм.

DOI: $10.21883 /$ FTT.2019.09.48109.16N

\section{1. Введение}

В рамках существующей классификации [1] принято различать внутренний (собственный) аномальный эффект Холла (АЭХ), определяемый поперечной добавкой к скорости за счет вклада фазы Берри в системах с сильным спин-орбитальным взаимодействием (СОВ), и внешний (примесный) АЭХ, связанный с рассеянием носителей заряда на примесных центрах. Однако, АЭХ возникает и в неколлинеарных ферромагнетиках, в которых ненулевая скалярная киральность $S_{i}\left(S_{j} \times S_{k}\right) \neq 0$ приводит к появлению эффективного магнитного поля даже в отсутствии СОВ [2], и в магнитных металлах с нетривиальной топологией спиновых структур в координатном пространстве [3-7].

При интерпретации экспериментальных данных возникает проблема идентификации механизмов АЭХ [1]. Асимметричное рассеяние (skew scattering), для которого угол рассеяния зависит от взаимной ориентации спина носителя заряда и магнитного момента примеси, дает зависимость $\rho_{x x}^{\mathrm{A}} \sim \rho_{x x}$ (случай чистых металлов $\left.\rho_{x x}<1 \mu \Omega \cdot \mathrm{cm}\right)$ [8]. Для диапазона сопротивлений $\rho_{x x}=1-100 \mu \Omega \cdot \mathrm{cm}$ доминирует внутренний АЭХ, обусловленный влиянием фазы Берри $\left(\rho_{x y}^{\mathrm{A}} \sim \rho_{x x}^{2}\right)$ [9]. Вкладом рассеяния за счет бокового смещения электрона (side jumping) [10] с аналогичным скейлингом $\left(\rho_{x y}^{\mathrm{A}} \sim \rho_{x x 0}^{2}\right.$, где $\rho_{x x 0}-$ остаточное сопротивление металла), как правило, пренебрегают [1]. „Грязный“ предел $\left(\rho_{x x}>100 \mu \Omega \cdot \mathrm{cm}\right)$ характеризуется промежуточным поведением $\rho_{x y}^{\mathrm{A}} \sim \rho_{x x}^{n}$ с показателями степени $n=1.6-1.8$, которые связывают с переходом к режиму прыжковой проводимости [1].

Важным примером „нарушения“ существующей классификации является смена режимов АЭХ в киральных магнетиках $\mathrm{Mn}_{1-x} \mathrm{Fe}_{x} \mathrm{Si}$, проявляющаяся в переходе от внутреннего эффекта, определяемого вкладом фазы Берри в фазе с магнитным порядком $\left(\rho_{x y}^{\mathrm{A}} \sim \rho_{x x}^{2}\right.$, $T<T_{c}$ ) [11], к внешнему, наблюдаемому в парамагнитном состоянии $\left(\rho_{x y}^{\mathrm{A}} \sim \rho_{x x}, T>T_{c}\right)[12,13]$. Необычная асимптотика реализуется в режиме доминирующего рассеяния носителей заряда $\left(\rho_{x x} \sim 10-100 \mu \Omega \cdot \mathrm{cm}\right)$ вне области применимости классической модели асимметричного рассеяния [8]. Линейный скейлинг АЭХ, зарегистрированный в парамагнитной фазе $\mathrm{Mn}_{1-x} \mathrm{Fe}_{x} \mathrm{Si}$ в широком диапазоне концентраций железа (до 30 at.\%) в умеренных магнитных полях (до 1.5 Т) при температурах до $60 \mathrm{~K}$, не имеет объяснения в рамках существующих моделей [1].

Количественный анализ параметров двух групп носителей заряда в ряду $\mathrm{Mn}_{1-x} \mathrm{Fe}_{x} \mathrm{Si}$ показал, что рост концентрации дырок с увеличением содержания железа 
приводит к сильному изменению вкладов в косвенное обменное взаимодействие между ионами марганца в первой и второй координационных сферах [13]. При этом режим наибольшей фрустрации реализуется в квантовых критических точках $\left(x^{*} \sim 0.12\right.$ и $\left.x_{\mathrm{C}} \sim 0.24\right)$, в которых амплитуды антиферромагнитного и ферромагнитного обмена между ионами марганца в различных координационных сферах становятся сопоставимы. В такой ситуации важно выяснить а) сохраняется ли асимптотика $\rho_{x y}^{\mathrm{A}} \sim \rho_{x x}$ для богатых железом составов $\mathrm{Mn}_{1-x} \mathrm{Fe}_{x} \mathrm{Si}$ и б) является ли линейный скейлинг аномального холловского сопротивления универсальным для систем с различными видами магнитной фрустрации [2].

\section{2. Методика эксперимента}

Для выяснения природы альтернативного механизма АЭХ в качестве объектов исследования были выбраны сильно коррелированные электронные системы с различными видами магнитной фрустрации (геометрической - в $\mathrm{Ho}_{1-x} \mathrm{Lu}_{x} \mathrm{~B}_{12}$, координационной - в $\mathrm{Mn}_{1-x} \mathrm{Fe}_{x} \mathrm{Si}$ и амплитудной - в $\left.\mathrm{Eu}_{1-x} \mathrm{Gd}_{x} \mathrm{~B}_{6}\right)$. Монокристаллические образцы высших боридов редкоземельных металлов были выращены методом бестигельной индукционной зонной плавки в атмосфере аргона в ИПМ НАНУ. Образец FeSi был вырезан из монокристаллического стержня, выращенного методом Чохральского и ранее использованного для подготовки образцов, исследованных в работах $[14,15]$. Вырезанные образцы шлифовались до необходимых размеров (толщины $100-500 \mu \mathrm{m})$ с последующим травлением поверхности в смеси кислот. Измерения гальваномагнитных свойств проводились при помощи стандартного шестиконтактного метода на экспериментальной установке ИОФ РАН в диапазоне температур 2-300 K в магнитном поле до 8 Т. Для анализа АЭХ использовались данные намагниченности, измеренные на СКВИД-магнитометре MPMS-5 (ИОФ РАН). Влияние размагничивающего поля учитывалось при помощи стандартной процедуры. Для сравнительного анализа использовались данные исследования АЭХ в твердых растворах замещения $\mathrm{Mn}_{1-x} \mathrm{Fe}_{x} \mathrm{Si}$ $(0 \leq x<0.3)$, ранее опубликованные в $[12,13]$.

\section{3. Результаты и обсуждение}

Исследования эффекта Холла в киральных магнетиках $\mathrm{Mn}_{1-x} \mathrm{Fe}_{x} \mathrm{Si}(0 \leq x \leq 0.3)$ [13] показали, что рост концентрации растворенного железа приводит к инверсии знаков аномального и нормального эффектов Холла, при этом в парамагнитной фазе $\mathrm{Mn}_{1-x} \mathrm{Fe}_{x} \mathrm{Si}$ линейный скейлинг $\rho_{x y}^{\mathrm{A}} \sim \rho_{x x}$ сохраняется во всем диапазоне исследованных температур (вплоть до $60 \mathrm{~K}$ ). Для узкозонного полупроводника $\mathrm{FeSi}$ знаки нормального $\left(\rho_{x y}>0\right)$ и аномального $\left(\rho_{x y}^{\mathrm{A}}<0\right)$ холловского сопротивления в $\mathrm{FeSi}$ хорошо согласуются со знаками соответствующих параметров, определяющих эффект
Холла в твердых растворах замещения $\mathrm{Mn}_{1-x} \mathrm{Fe}_{x} \mathrm{Si}$ с большим содержанием железа $(x>0.12)$ [13]. Однако, в случае $\mathrm{FeSi}$ амплитуда АЭХ монотонно растет с понижением температуры более чем на 6 порядков по абсолютной величине (от $\rho_{x y}^{\mathrm{A}} \approx-0.17 \mu \Omega \cdot \mathrm{cm}$ при $60 \mathrm{~K}$ до $\rho_{x y}^{\mathrm{A}} \approx-0.7 \Omega \cdot \mathrm{cm}$ при $1.8 \mathrm{~K}$, см. рис. 1$)$. Сильную температурную зависимость, по-видимому, следует связать с особенностями спин-поляронного режима проводимости, определяющего зарядовый транспорт в FeSi при температурах ниже $80 \mathrm{~K}$ [14]. Более того, в отличие от $\mathrm{Mn}_{1-x} \mathrm{Fe}_{x} \mathrm{Si}$, в парамагнитной фазе моносилицида железа $\left(T>T_{C}=15 \mathrm{~K}[14]\right)$ доминирует аномальный вклад, определяемый изменением фазы Берри $\left(\rho_{x y}^{\mathrm{A}} \sim \rho_{x x}^{2}\right.$, вставка на рис. 1$)$. Наблюдаемое различие обусловлено особенностями спин-поляронных состояний, формирование которых сопровождается полной спиновой поляризацией ионов железа в окрестности носителя заряда $p$-типа с характерным радиусом поляризованной области 4-5 [14]. При этом возникновение необычного скейлинга $\rho_{x y}^{\mathrm{A}} \sim \rho_{x x}^{1.5}$ (вставка на рис. 1) ниже $15 \mathrm{~K}$, по-видимому, определяется переходом к основному магнитному состоянию $\mathrm{FeSi}$, в котором аномальный эффект Холла характеризуется сильным гистерезисом за счет появления легкой оси намагничивания вдоль кристаллографического направления 〈111〉 [14].

В отличие от моносилицида железа, в исследованных высших боридах редкоземельных металлов $\mathrm{Ho}_{0.5} \mathrm{Lu}_{0.5} \mathrm{~B}_{12}$ и $\mathrm{Eu}_{1-x} \mathrm{Gd}_{x} \mathrm{~B}_{6}(x<0.04)$ аномальный вклад в эффект Холла $\rho_{x y}^{\mathrm{A}} \sim \rho_{x x} M$, обусловленный асимметричным рассеянием носителей заряда, проявляется в парамагнитной фазе (рис. 2-3). Однако, прямой аналогии с эффектом Холла в $\mathrm{Mn}_{1-x} \mathrm{Fe}_{x} \mathrm{Si}$ не наблюдается. Действительно, для $\mathrm{Ho}_{0.5} \mathrm{Lu}_{0.5} \mathrm{~B}_{12}$ в магнитных полях выше границы антиферромагнитной фазы полевая зависимость холловского сопротивления с хорошей точностью описывается выражением $\rho_{x y}=R_{H} B+S_{1} \rho_{x x} M$ (результат аппроксимации

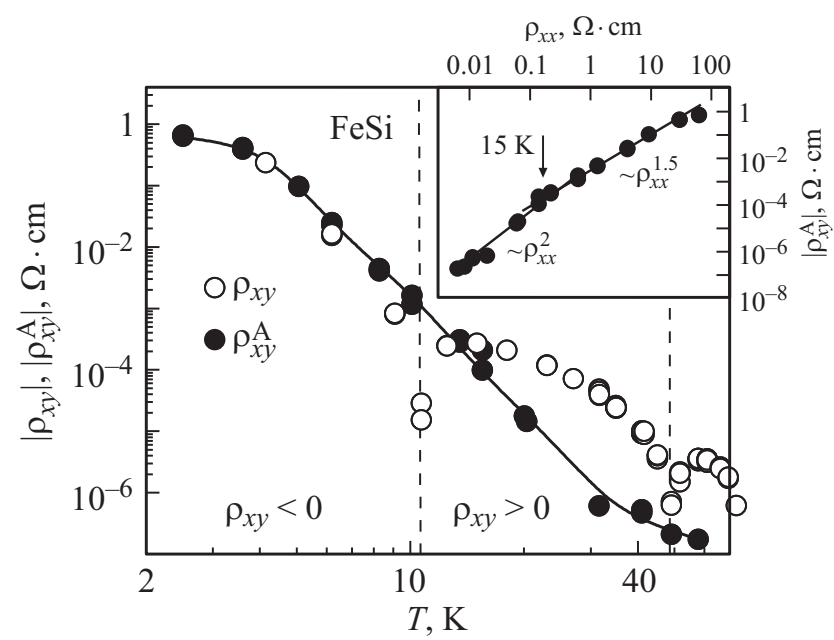

Рис. 1. Температурные зависимости холловского сопротивления $\rho_{x y}$ и аномального вклада в эффект Холла $\rho_{x y}^{\text {А }}$ для $\mathrm{FeSi}$ в магнитном поле 7 T. Для всего диапазона температур $\rho_{x y}^{\mathrm{A}}<0$. На вставке показана параметрическая зависимость $\rho_{x y}^{\mathrm{A}}=f\left(\rho_{x x}\right)$. 


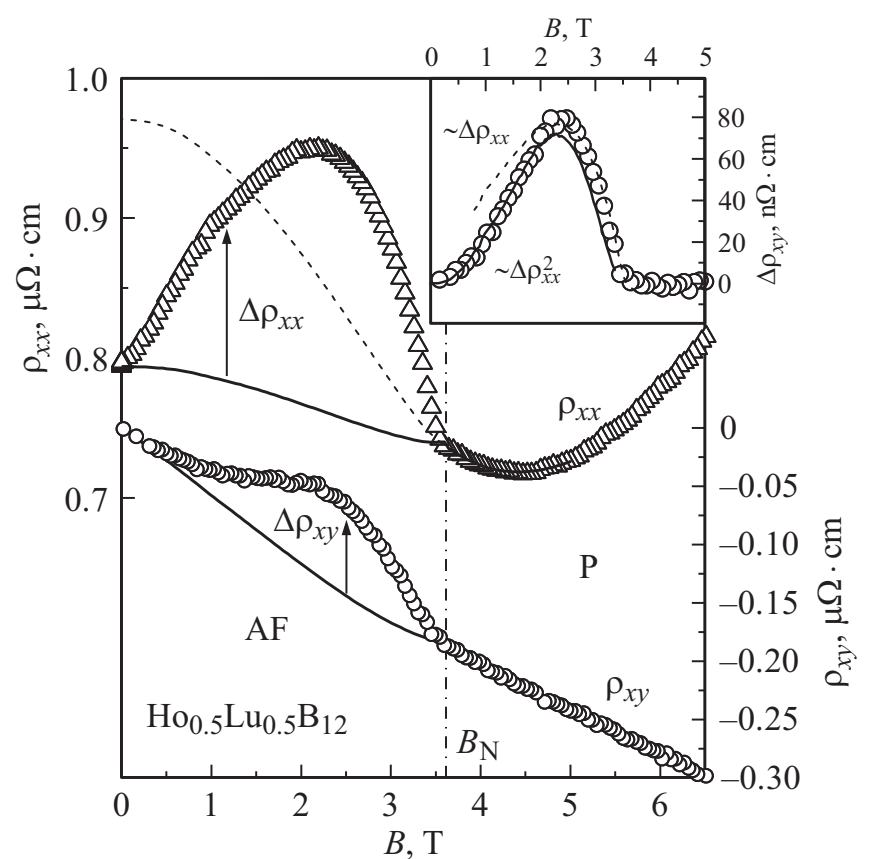

Рис. 2. Полевые зависимости удельного сопротивления $\rho_{x x}$ и холловского сопротивления $\rho_{x y} \quad \mathrm{~B}^{\mathrm{H}} \mathrm{Ho}_{0.5} \mathrm{Lu}_{0.5} \mathrm{~B}_{12}$ (температура Нееля $T_{\mathrm{N}} \approx 3.4 \mathrm{~K}$ ) при температуре $2.1 \mathrm{~K}$ (магнитное поле направлено вдоль $\langle 100\rangle)$. На вставке показан топологический вклад в холловское сопротивление (см. текст).

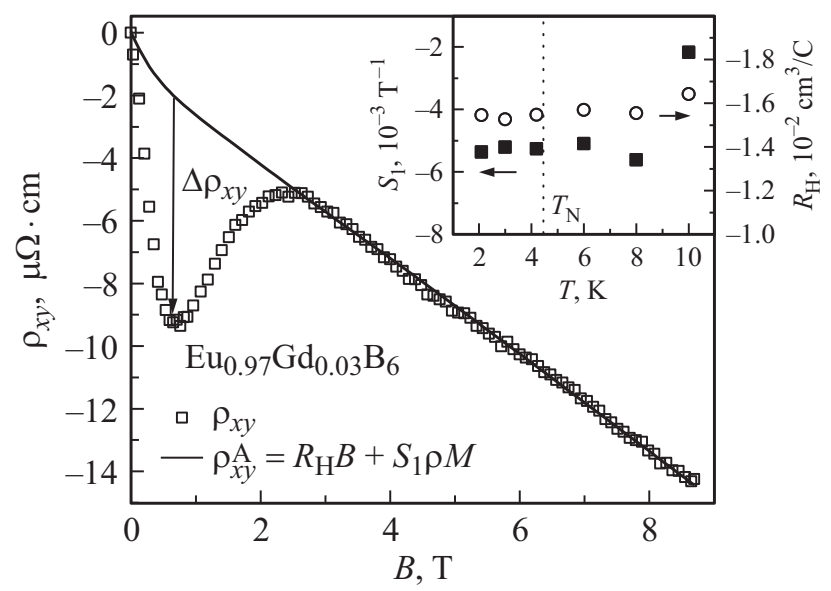

Рис. 3. Эффект Холла в $\mathrm{Eu}_{0.97} \mathrm{Gd}_{0.03} \mathrm{~B}_{6}$ при $T=2.1 \mathrm{~K}$. Линией обозначен аномальный вклад $\rho_{x y}^{\mathrm{A}}=R_{\mathrm{H}} B+S_{1} \rho_{x x} M$ $\left(R_{H}=-1.55 \cdot 10^{-2} \mathrm{~cm}^{3} / \mathrm{C}\right.$ и $\left.S_{1}=-5.2 \cdot 10^{-3} \mathrm{~T}^{-1}\right)$. Стрелкой показан топологический эффект Холла $\Delta \rho_{x y}$. На вставке представлена температурная эволюция параметров $S_{1}$ и $R_{H}$ $\left(T_{\mathrm{N}} \approx 4.3 \mathrm{~K}-\right.$ температура Нееля).

с параметрами $R_{H}=-3.74 \cdot 10^{-4} \mathrm{~cm}^{3} /$ С и $S_{1}=3.1 \mathrm{~T}^{-1}$ представлен сплошной линией на рис. 2). Отметим, что нормальный коэффициент Холла в пределах точности $(\sim 10 \%)$ не зависит от ориентации магнитного поля относительно кристаллографических направлений, в то время как для параметра $S_{1}$ наблюдается сильная анизотропия (со сменой знака для направления $\langle 111\rangle$ ) и сильная зависимость от температуры в окрестности точки Нееля для всех исследованных образцов. Так, для $B \|[110]$ параметр $S_{1}=-(0.7 \pm 0.1) \mathrm{T}^{-1}$ практически не зависит от температуры, в то время как для $B \|[111] \rho_{x y}^{\mathrm{A}}$ меняет знак, уменьшаясь от значений $S_{1}=+4.7 \mathrm{~T}^{-1}$ при $T=2 \mathrm{~K}$ до $S_{1}=-5.1 \mathrm{~T}^{-1}$ при $T=4.2 \mathrm{~K}$.

C другой стороны, в антиферромагнитной фазе $\mathrm{Ho}_{0.5} \mathrm{Lu}_{0.5} \mathrm{~B}_{12}$ экспериментальные данные $\rho_{x y}(B)$ заметно отклоняются от расчетной кривой в сторону положительных значений (рис. 2). Амплитуда разности $\Delta \rho_{x y}=\rho_{x y}-\left(R_{H} B+S_{1} \rho_{x x} M\right)$ немонотонно меняется с ростом магнитного поля, проходя через максимальные значения $\Delta \rho_{x y} \sim+80 \mathrm{n} \Omega \cdot \mathrm{cm}$ в поле $B^{*} \approx 2.5 \mathrm{~T}$ при $2.1 \mathrm{~K}$. Более того, амплитуда $\Delta \rho_{x y}$ зависит от ориентации магнитного поля и варьируется в пределах от $\Delta \rho_{x y} \approx+80 \mathrm{n} \Omega \cdot \mathrm{cm}(B \|[100])$ до $\Delta \rho_{x y} \approx+15 \mathrm{n} \Omega \cdot \mathrm{cm}$ $(B \|[111])$ при $T=2 \mathrm{~K}$. На полевых зависимостях магнитосопротивления (верхняя часть рис. 2) наблюдается аналогичная аномалия, амплитуда которой уменьшается по мере приближения к точке Нееля. Для выделения магнитного вклада в удельное сопротивление экспериментальные данные $\rho_{x x}(B)$ аппроксимировались выражением $\rho_{x x}=\rho_{x x 0}+A B^{2}-C M^{2}$, в котором второе слагаемое $\left(\sim B^{2}\right)$ определяет вклад зонных электронов, а третий член $\left(\sim M^{2}\right)$ учитывает отрицательное магнитосопротивление (OMC) за счет магнитного рассеяния носителей заряда на локализованных магнитных моментах. Предполагалось, что в антиферромагнитной фазе магнитный порядок уменьшает вклад ОМС $\left(C\left(B<B_{C}\right)<C\left(B>B_{C}\right)\right.$ для $\left.T=2.1 \mathrm{~K}\right)$, в то время как зонный вклад остается неизменным. Результирующий разностный вклад в удельное сопротивление $\Delta \rho_{x x}$ представлен в верхней части рис. 2. Сопоставление магнитных вкладов в удельное сопротивление $\Delta \rho_{x x}$ и эффект Холла $\Delta \rho_{x y}$ показало, что в области полей $B<B^{*}$ выполняется соотношение $\Delta \rho_{x y} \sim\left(\Delta \rho_{x x}\right)^{2}$, а в области полей $B>B^{*}$ магнитный вклад в эффект Холла с лучшей точностью описывается соотношением $\Delta \rho_{x y} \sim \Delta \rho_{x x}$ (вставка на рис. 2). Необычная корреляция между магнитными вкладами, на наш взгляд, может возникать изза экзотической спиновой структуры в антиферромагнитной фазе $\mathrm{Ho}_{0.5} \mathrm{Lu}_{0.5} \mathrm{~B}_{12}$ по аналогии с топологическим эффектом Холла в $\mathrm{MnSi}$ и родственных системах.

В случае твердых растворов замещения $\mathrm{Eu}_{1-x} \mathrm{Gd}_{x} \mathrm{~B}_{6}$ $(x<0.04)$ линейный скейлинг аномального холловского сопротивления наблюдается в случае легированных составов. В основном состоянии $\mathrm{Eu}_{1-x} \mathrm{Gd}_{x} \mathrm{~B}_{6}$ знак АЭХ определяется типом магнитного порядка $\left(S_{1}>0\right.$ для ферромагнитной и $S_{1}<0$ для антиферромагнитной фазы). Наиболее интересная ситуация реализуется в $\mathrm{Eu}_{0.97} \mathrm{Gd}_{0.03} \mathrm{~B}_{6}$ (рис. 3), для которого параметры $R_{H}$ и $S_{1}$ в выражении для холловского сопротивления $\rho_{x y}=R_{H} B+S_{1} \rho_{x x} M$ не зависят от температуры в диапазоне от $2 \mathrm{~K}$ до $8 \mathrm{~K}$ (вставка на рис. 3). При этом, как и в случае додекаборидов, в малых магнитных полях зарегистрирован дополнительный вклад в холловское сопротивление, уменьшающийся по амплитуде с ростом температуры по мере приближения к точке Нееля. Амплитуда вклада достигает значений $\Delta \rho_{x y}=-7.5 \mu \Omega \cdot \mathrm{cm}$ 


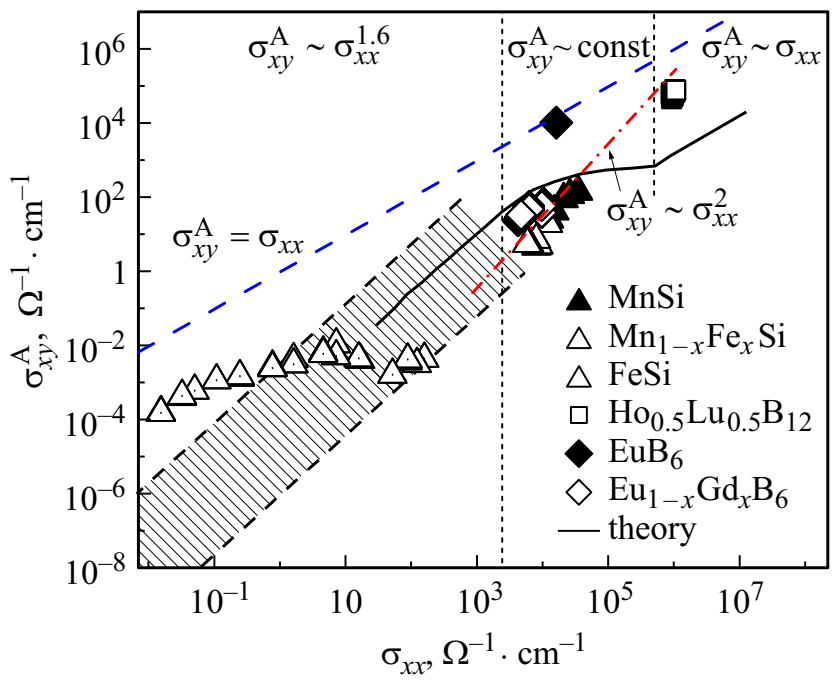

Рис. 4. Параметрические зависимости $\sigma_{x y}^{\mathrm{A}}=f\left(\sigma_{x x}\right)$ для $\mathrm{Ho}_{0.5} \mathrm{Lu}_{0.5} \mathrm{~B}_{12}, \quad \mathrm{Mn}_{1-x} \mathrm{Fe}_{x} \mathrm{Si} \quad(0 \leq x \leq 1) \quad$ и $\quad \mathrm{Eu}_{1-x} \mathrm{Gd}_{x} \mathrm{~B}_{6}$ $(x<0.04)$. Линии и заштрихованная область соответствуют предсказаниям модели Оноды с соавторами [17].

при $2.1 \mathrm{~K}$ (рис. 3). Однако, в отличии от $\mathrm{Ho}_{0.5} \mathrm{Lu}_{0.5} \mathrm{~B}_{12}$ в случае $\mathrm{Eu}_{1-x} \mathrm{Gd}_{x} \mathrm{~B}_{6}$ корреляций с поведением магнитосопротивления не наблюдается.

Таким образом, несмотря на то, что линейная асимптотика наблюдается для различных твердых растворах замещения $\left(\mathrm{Ho}_{0.5} \mathrm{Lu}_{0.5} \mathrm{~B}_{12}, \mathrm{Mn}_{1-x} \mathrm{Fe}_{x} \mathrm{Si}(0 \leq x \leq 0.3)\right.$ и $\left.\mathrm{Eu}_{1-x} \mathrm{Gd}_{x} \mathrm{~B}_{6}(x<0.04)\right)$, полученные данные не позволяют сделать однозначный вывод о единой природе аномального эффекта Холла с линейным скейлингом $\rho_{x y}^{\mathrm{A}} \sim \rho_{x x}$, определяемым асимметричным рассеянием носителей заряда. Более того, сопоставление полученных в работе данных с предсказаниями теоретической работы Оноды с соавторами [16] показывает, что для всех диапазонов значений удельного сопротивления с различными скейлинговыми соотношениями наблюдается существенное отклонение экспериментальных данных от модельных зависимостей. Наиболее сильно это отклонение проявляется для $\mathrm{Ho}_{0.5} \mathrm{Lu}_{0.5} \mathrm{~B}_{12}$ (высокие значения проводимости), $\mathrm{EuB}_{6}$ (промежуточный диапазон) и $\mathrm{FeSi}$ (режим сильного примесного рассеяния), см. рис. 4. С другой стороны, линейная аппроксимация экспериментальных точек для легированных составов дает зависимость, близкую к $\sigma_{x y} \sim\left(\sigma_{x x}\right)^{2}$, что в пересчете на удельное сопротивление эквивалентно $\rho_{x y} \sim \operatorname{const}\left(\rho_{x x}\right)$. Подобная зависимость ранее не наблюдалась и не имеет объяснения в рамках существующих моделей.

\section{4. Заключение}

Результаты исследования показали, что во всех исследованных в работе системах $\mathrm{Ho}_{0.5} \mathrm{Lu}_{0.5} \mathrm{~B}_{12}, \mathrm{Mn}_{1-x} \mathrm{Fe}_{x} \mathrm{Si}$ $(0 \leq x \leq 0.3)$ и $\mathrm{Eu}_{1-x} \mathrm{Gd}_{x} \mathrm{~B}_{6}(x<0.04)$ с разным типом фрустрации в парамагнитной фазе наблюдается вклад в аномальный эффект Холла, пропорциональный сопротивлению $\left(\rho_{x y}^{\mathrm{A}} \sim \rho_{x x}\right)$. Полученные данные указывают на необходимость разработки теоретических моделей, корректно описывающих вклад асимметричного рассеяния носителей заряда в эффект Холла для систем с неколлинеарными спиновыми структурами.

\section{Благодарности}

Авторы выражают признательность И.И. Лобановой за предоставление данных измерений транспортных свойств $\mathrm{Mn}_{1-x} \mathrm{Fe}_{x} \mathrm{Si}(0 \leq x<0.3)$ и полезные обсуждения.

\section{Финансирование работы}

Работа выполнена при финансовой поддержке Российского фонда фундаментальных исследований (проект 18-02-01152) и Российского научного фонда (проект 17-12-01426, исследование магнитосопротивления в $\left.\mathrm{Ho}_{0.5} \mathrm{Lu}_{0.5} \mathrm{~B}_{12}\right)$.

\section{Конфликт интересов}

Авторы заявляют, что у них нет конфликта интересов.

\section{Список литературы}

[1] N. Nagaosa, J. Sinova, S. Onoda, A.H. MacDonald, N.P. Ong. Rev. Mod. Phys., 82, 1539 (2010).

[2] C.D. Batista, S.-Z. Lin, S. Hayami, Y. Kamiya. Rep. Prog. Phys. 79, 084504 (2016).

[3] Y. Taguchi, Y. Oohara, H. Yoshizawa, N. Nagaosa, Y. Tokura. Science 291, 2573 (2001).

[4] P. Pureur, F. Wolff Fabris, J. Schaf, I.A. Campbell. Europhys. Lett. 67, 123 (2004).

[5] A. Neubauer, C. Pfleiderer, B. Binz, A. Rosch, R. Ritz, P.G. Niklowitz, P. Böni. Phys. Rev. Lett. 102, 186602 (2009).

[6] Y. Machida, S. Nakatsuji, S. Onoda, T. Tayama, T. Sakakibara. Nature 463, 210 (2010).

[7] Z.H. Liu, Y.J. Zhang, G.D. Liu, B. Ding, E.K. Liu, H.M. Jafri, Z.P. Hou, W.H. Wang, X.Q. Ma, G.H. Wu. Sci. Rep. 7, 515 (2017).

[8] J. Smit. Physica 24, 1, 39 (1958).

[9] T. Jungwirth, Q. Niu, A.H. MacDonald. Phys. Rev. Lett. 88, 207208 (2002).

[10] L. Berger. Phys. Rev. B 2, 4559 (1970).

[11] M. Lee, Y. Onose, Y. Tokura, N.P. Ong. Phys. Rev. B 75, 172403 (2007).

[12] V.V. Glushkov, I.I. Lobanova, V.Yu. Ivanov, S.V. Demishev. Письма в ЖЭТФ 101, 512 (2015).

[13] V.V. Glushkov I.I. Lobanova, V.Yu. Ivanov, V.V. Voronov, V.A. Dyadkin, N.M. Chubova, S.V. Grigoriev, S.V. Demishev. Phys. Rev. Lett. 115, 256601 (2015).

[14] В.В. Глушков, И.Б. Воскобойников, С.В. Демишев, И.В. Кривицкий, А. Меновски, В.В. Мещалков, Н.А. Самарин, Н.Е. Случанко. ЖЭТФ 126, 2, 444 (2004).

[15] V.V. Glushkov, B.P. Gorshunov, E.S. Zhukova, S.V. Demishev, A.A. Pronin, N.E. Sluchanko, S. Kaiser, M. Dressel. Phys. Rev. B 84, 7, 073108 (2011).

[16] S. Onoda, N. Sugimoto, N. Nagaosa. Phys. Rev. B 77, 16, 165103 (2008).

Редактор Т.Н. Василевская 